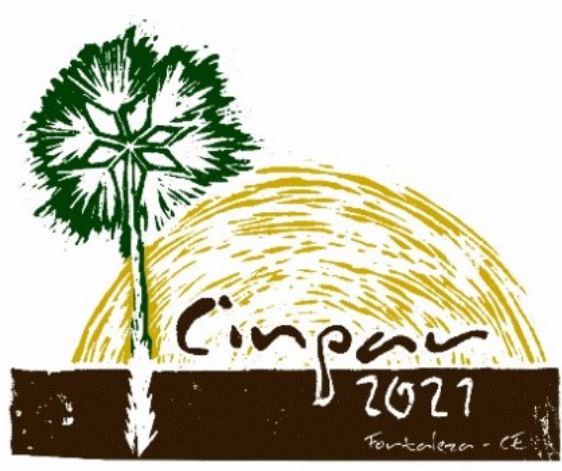

XVII Congresso Internacional sobre Patologia e

Reabilitação das Construções

XVII Congreso Internacional sobre Patología y Rehabilitación de las Construcciones

XVII International Conference on Pathology and Constructions Rehabilitation

FORTALEZA (Brasil), 3 a 5 de junho de 2021

https://doi.org/10.4322/CINPAR.2021.075

\title{
Avaliação das características do sistema de revestimento de fachadas no processo de degradação por meio do Método de Mensuração de Degradação
}

\section{Evaluation of the characteristics of the facade cladding system in the degradation process using the Degradation Measurement Method}

\author{
Suzanna Ferreira PONTES ${ }^{1}$, Jéssica Siqueira de SOUZA ${ }^{2}$, Carla Bozzi PIAZZAROLLO ${ }^{3}$, Alan Ribeiro SANTOS ${ }^{4}$ \\ ${ }^{1}$ Universidade de Brasília, Brasília, Brasil, suzanna.pontes14@hotmail.com \\ ${ }^{2}$ Universidade de Brasília, Brasília, Brasil, jss.siqueira@gmail.com \\ ${ }^{3}$ Universidade de Brasília, Brasília, Brasil, carlabp@gmail.com \\ ${ }^{4}$ Universidade de Brasília, Brasília, Brasil, alanrsmbs@gmail.com
}

\begin{abstract}
Resumo: A vida útil é um período de tempo que abrange desde o início do uso de um produto até o momento que o desempenho é inferior ao preestabelecido. O desempenho é comprometido pela degradação, pois quanto maior for o nível de degradação menor será o desempenho e, consequentemente, menor a durabilidade do edifício e seus sistemas. Este artigo visa analisar o impacto das características dos revestimentos de fachada sobre a vida útil através do Método de Mensuração de Degradação. A metodologia consiste na quantificação de degradação por meio de mapeamento e quantificação dos danos. Este método permite realizar diversas análises relacionadas às condições de degradação e vida útil dos sistemas. Dentre as análises possíveis, esta pesquisa avalia as a intensidade e a probabilidade de ocorrência de degradação em diferentes zonas da fachada (paredes contínuas, aberturas, sacadas, cantos e extremidades, transição entre pavimentos e topo) por meio de curvas da probabilidade de danos, assim possibilitando a identificação das zonas mais sensíveis à degradação. Os resultados demonstraram que as paredes contínuas e os cantos e extremidades são as zonas mais propensas a degradação e o descolamento cerâmico como o dano mais incidente em sistemas de revestimento cerâmico.
\end{abstract}

Palavras-chave: Durabilidade; Desempenho; Vida útil; Degradação; Fachada.

Abstract: The service life is a period of time that extends from the beginning of the use of a product until the moment when the performance is inferior to the pre-established. Performance is compromised by degradation, because the higher the level of degradation, the lower the performance and, consequently, the less the durability of the building and its systems. This article aims to analyze the impact of the characteristics of facade coverings on the useful life through the Degradation Measurement Method. The methodology consists of quantifying degradation by mapping and quantifying damage. This method allows to perform several analyzes related to the degradation conditions and service life of the systems. Among the possible analyzes, this research assesses the intensity and the probability of degradation occurring in different areas of the facade (continuous walls, openings, balconies, corners and ends, transition between floors and top) through curves of the probability of damage, thus enabling the identification of the areas most sensitive to degradation. The results showed that the continuous walls and the corners and ends are the areas most prone to degradation and ceramic detachment as the most incident damage in ceramic coating systems.

Keywords: Durability; Performance; Service life; Degradation; Facade. 


\section{Introdução}

O sistema de revestimento de fachada do edifício é um dos elementos que compõe o edifício mais expostos às condições desfavoráveis, principalmente devido à constante interação com o meio ambiente. As solicitações mais comuns as quais são submetidos o revestimento são: variações térmicas, ações de vento, ações de umidade, carregamentos estáticos e dinâmicos, ações de chuvas, deformações diferenciais, ações de peso próprio, abrasão, impactos e umidade do solo (Silva, 2006; Sousa, 2008). Estas solicitações permitem o surgimento dos primeiros indícios de degradação.

A crescente degradação compromete o desempenho do sistema, o que acarreta na diminuição de sua vida útil, uma vez que a vida útil está diretamente associada ao desempenho que o sistema foi projetado para atender. Para que cumpram suas funções, as fachadas devem conservar as suas características durante um determinado período, de modo que supram às necessidades e expectativas dos usuários (Bauer et al., 2020).

O processo de degradação deve ser tratado no início, quando os danos ainda são pequenos, caso contrário, poderá implicar em elevados custos de reparo no futuro, além da evolução de danos leve para problemas físicos reais da fachada (Bauer et al., 2015). A observação sistemática dos danos e do sistema de fachada auxilia na elaboração de um diagnóstico mais eficaz, uma vez que esta observação permite compreender melhor os mecanismos de degradação e estabelece hipóteses de causa e efeito dos danos (Silvestre, 2005). Estas observações garantem que a fachada atenda a qualidade e funcionalidade exigida pelos usuários. A importância desta pesquisa se deve, portanto, a crescente necessidade de se prolongar o tempo em serviço do sistema, aumentando a vida útil e otimizando os custos relacionados à manutenção.

Diante do exposto, juntamente com a crescente necessidade de estudos que visam aprofundar o conhecimento sobre degradação e durabilidade do sistema de revestimento cerâmico em fachadas, este artigo visa analisar o impacto das características de cada zona dos revestimentos de fachada sobre a vida útil. Por meio da aplicação do Método de Mensuração de Degradação (MMD) que tem como base a quantificação da degradação através de indicadores de degradação, foi possível definir e entender quais as zonas de fachadas de edifícios são mais suscetíveis à degradação e identificar as possíveis causas. Os danos mais freqüentes e mais avaliados em sistema de revestimento cerâmico de edifícios localizados no Distrito Federal, Brasil, são descolamento cerâmico, fissura, falha de rejunte e eflorescência. Esta análise conjunta e pormenorizada das zonas com os danos subsidiam informações para novos projetos e auxiliam nas ações de manutenção deste sistema.

\section{Metodologia}

A partir de uma base de dados desenvolvida por pesquisadores da Universidade de Brasília, foram estudados edifícios situados em Brasília, Brasil (Souza et al., 2019a; Bauer et al., 2020). A base de dados é constituída por mais de 45 edifícios com idades entre 5 e 50 anos e que representam mais de $80.000 \mathrm{~m}^{2}$ de fachada revestidas por cerâmicas. Esta base de dados foi obtida para o desenvolvimento do Método de Mensuração de Degradação (MMD) que tem como objetivo quantificar a degradação por meio de indicadores de degradação que permitem análises da vida útil de sistemas dos edifícios. A avaliação da degradação é baseada no MMD que é composto por diversas etapas, que envolvem desde a inspeção visual e fotográfica dos edifícios até a obtenção dos indicadores de degradação. A Figura 1 ilustra as principais etapas para a quantificação da degradação. 


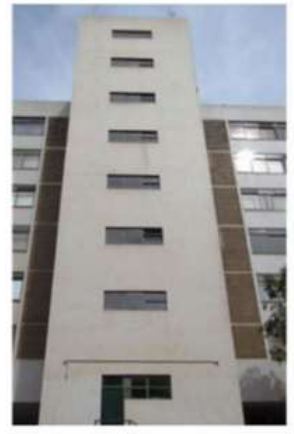

(a)

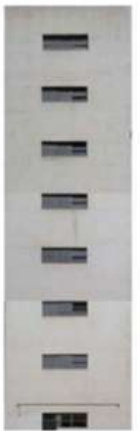

(b)

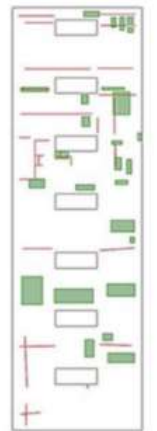

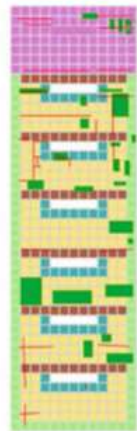

(d)

Figura 1 - Principais etapas do MMD para a quantificação da degradação. (a) Registro fotográfico; (b) Ortogonalização; (c) Mapeamento de danos e; (d) Definição das zonas (Souza, 2019)

Uma das grandes vantagens do MMD é a flexibilidade de aplicação e obtenção de analises, pois este método permite inúmeras formas de análises, desde as mais simples até as mais complexas. $O$ estudo do perfil de degradação que ocorre nas fachadas pode ser observado quando a fachada é dividida em zonas. As zonas adotadas pelo MMD são as paredes contínuas, aberturas, cantos e extremidades, sacadas, transição entre pavimentos e topo (Figura 2).

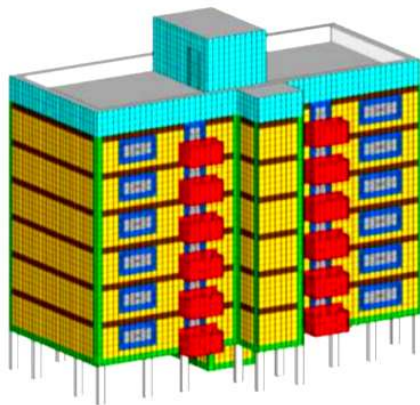

\author{
PC Paredes Contínuas \\ AB Aberturas \\ Sacadas \\ Cantos e Extremidades \\ Transição entre pavimentos \\ TO Topo
}

Figura 2 - Zonas constituintes da fachada (Souza ${ }^{1}$ et al., 2019)

Dentre os indicadores de degradação resultantes da aplicação do MMD, destaca-se o Fator de Danos (FD). Este indicador permite realizar análises preliminares de degradação, posto que este pode ser associado ao percentual de degradação das fachadas e pode ser aplicado tanto para os danos quanto para as zonas que compões as fachadas. Detsa ofrma, é possível realizar uma análise pormenorizada do processo de degradação dos sistemas. A Equação 1 descreve como o FD pode ser obtido quando aplicado para os tipos de danos. Quando aplicado às zonas, os danos são contabilizados para cada zona.

$$
F D=\frac{\sum A_{d(n)}}{A_{t}}
$$

Em que FD é o indicador de degradação Fator de Danos, $A_{d(n)}$ é a área de determinado dano (n) em $m^{2}$ e $A_{t}$ é a área total da amostra de fachada em $\mathrm{m}^{2}$.

Com os valores de FD obtidos com a base de dados, a obtenção de curvas de probabilidade de danos aplicadas às zonas tornou-se possível. Para isto, o software utilizado para gerar as curvas de probabilidade foi o EasyFit. As equações testadas foram Logística, Lognormal, Normal, Weibull e Student. A escolha da curva de melhor ajuste foi analisada considerando os testes de Komogorov, Anderson-Darling e ChiQuadrado.

\title{
3. Análise de resultados
}

A Figura 3 ilustra as curvas de probabilidade obtidas para cada zona considerando todos os danos existentes nas fachadas. 

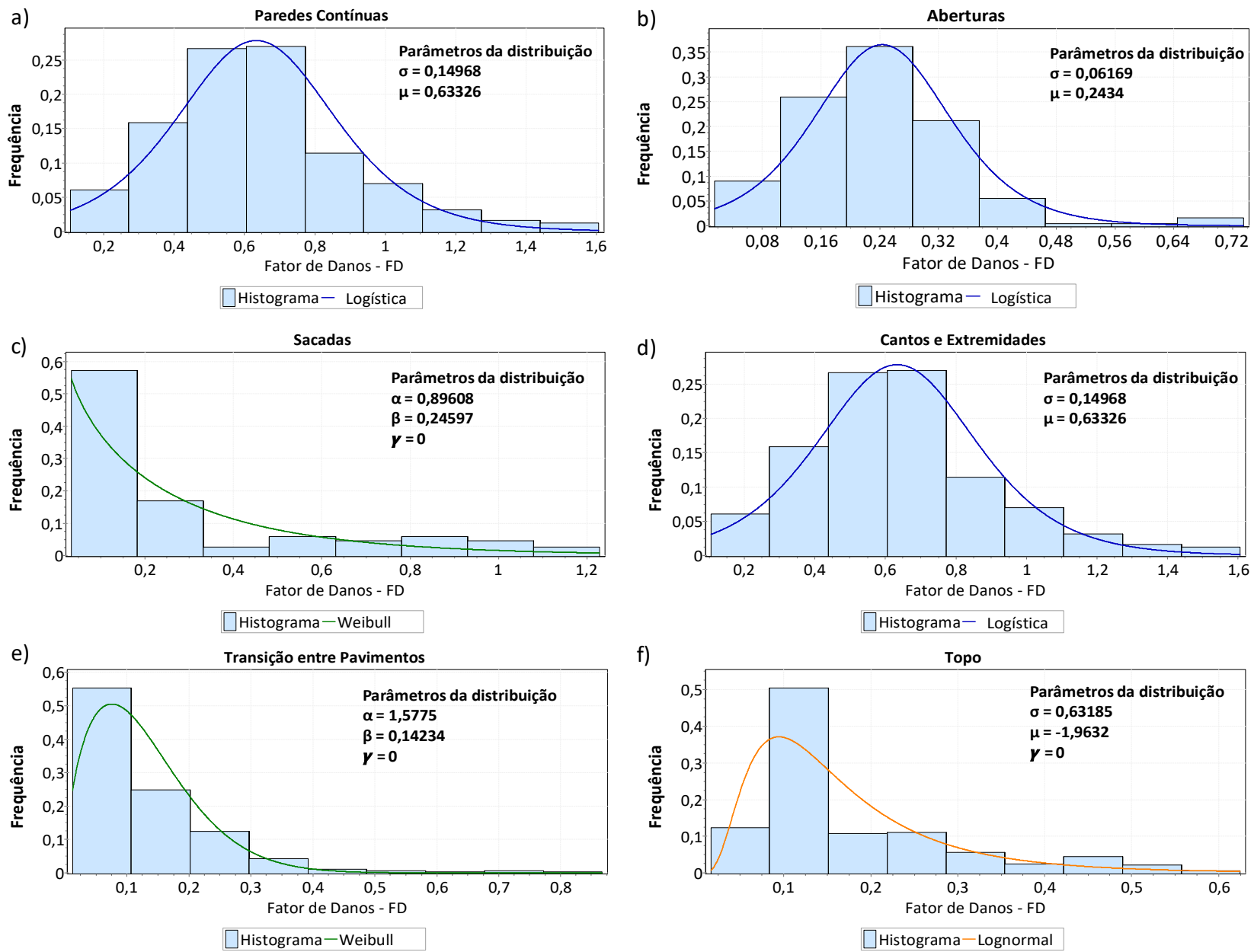

Figura 3 - Curvas de probabilidade para as zonas constituintes da fachada.

Analisando a Figura 3a nota-se que a zona de paredes contínuas (PC) apresentou, para a maior probabilidade da curva, um valor de FD igual a 0,650. Para avaliação semelhante à zona de aberturas (Figura 3b) apresentou valor igual a 0,240, a zona de sacadas (Figura 3c) igual a 0,025, a zona de cantos e extremidades (Figura 3d) igual a 0,650, a zona de transição entre pavimentos (Figura 3e) igual a 0,075 e zona de topo (Figura 3f) igual a 0,090.

As zonas PC e CE apresentam uma maior frequência de valores de FD elevados (0,4-0,8). Para as paredes contínuas esta constatação se deve, primeiramente, ao fato de esta zona possuir maior área em relação às demais e, por se tratar de uma zona que se situa em confinamento em relação às outras, há maior restrição da sua movimentação, dificultando a adaptação das deformações, tornando os danos maiores. Os resultados observados para os cantos e extremidades, por sua vez, possivelmente se devem a descontinuidade dessa zona, o que a leva a ter elevadas tensões, implicando em valores de FD maiores. Resultados similares foram obtidos por Souza et al.(2019b), que identificaram as zonas PC e CE como mais críticas para a ocorrência de danos, principalmente nos casos de edifícios mais antigos. A amplitude dos valores de FD para as zonas de paredes contínuas e cantos e extremidades foi maior do que para as demais zonas, o que mostra que tais zonas podem apresentar maiores áreas de degradação.

Embora as paredes contínuas e cantos e extremidades apresentem os maiores valores de FD, observa-se que as zonas de sacadas e transição entre pavimentos possuem maiores valores de probabilidade de ocorrer, que podem ser observados pelos pontos máximos da curva de probabilidade. Este fato tem relação com as particularidades de cada uma dessas zonas. As sacadas possuem uma elevada exposição a chuva, radiação solar e tensões devido a sua configuração em balanço, o que favorece o surgimento dos danos, no entanto, as sacadas apresentaram valores de FD menores, possivelmente devido apouca área disponível de sacadas dos edifícios analisados.Já na transição entre pavimentos é frequente a ausência de juntas de 
dilatação horizontal, o que interfere na dissipação das deformações causadas pelo sistema alvenaria/estrutura, expondo assim essa zona ao fenômeno da degradação.

A Figura 4 ilustra as curvas de probabilidade obtidas para cada tipo de dano considerando todas as zonas constituintes das fachadas.

a)

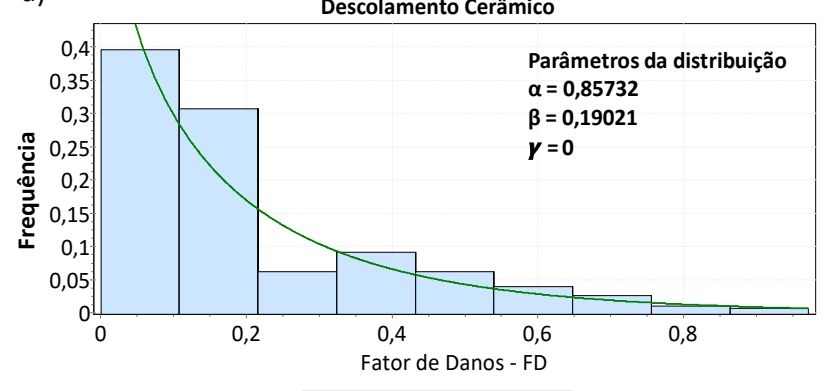

c)

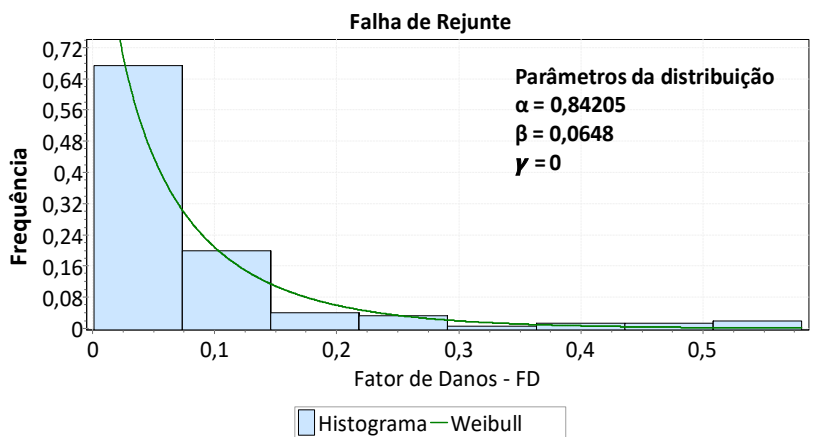

b)

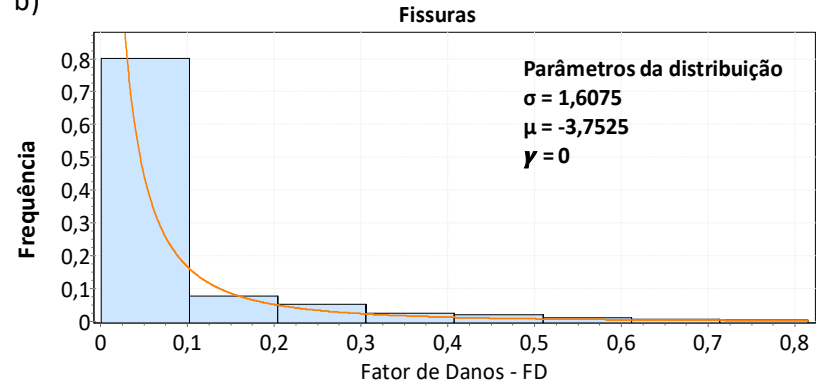

d)

$\square$ Histograma-Lognormal

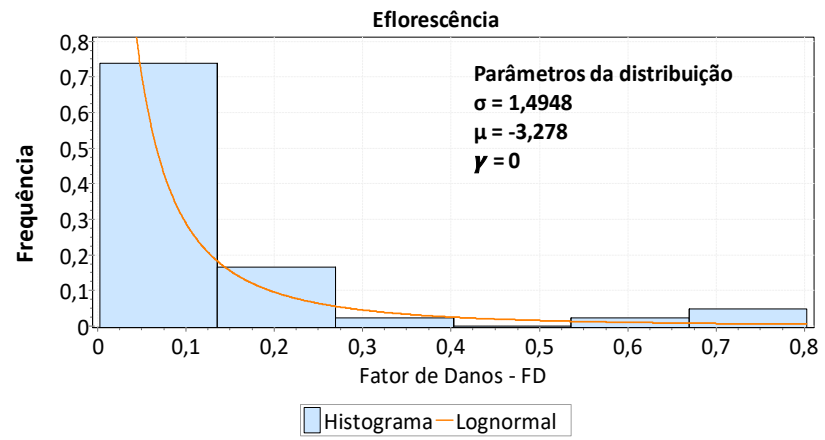

Figura 4 - Curvas de probabilidade para os danos existentes na fachada.

A maior probabilidade da curva para o descolamento cerâmico (Figura 4a) equivale a um valor de FD igual a 0,050, para as fissuras (Figura 4b) igual a 0,025, para a falha de rejunte (Figura 4c) igual a 0,025 e a eflorescência (Figura 4d) igual a 0,040. Em geral, todos os tipos de danos apresentam maior frequência de ocorrência nos valores de FD mais baixos, em sua maioria entre 0 e 0,3 . Para as falhas de rejunte e as fissuras, que praticamente não têm registros de ocorrência de danos além desta faixa, os resultados podem ter relação com a área total do tipo de dano registrada nas amostras analisadas em relação a área total. $\mathrm{A}$ distribuição dos valores de FD nas classes do histograma chama atenção para o descolamento cerâmico, dano que registrou os maiores valores de FD. O resultado observado pode ser relacionado a falhas de assentamento das placas cerâmicas, falta de juntas de movimentação e uso de rejunte rígido, comprometendo a aderência da placa cerâmica quando submetida à excessiva movimentação dos edifícios. As eflorescências apresentam uma classe com valores entre 0,7 e 0,8, o que pode estar associado com a idade dos edifícios avaliados e até mesmo com algum problema ou erro de execução do sistema. Estes resultados vão ao encontro do observado por Souza et al. (2019c) e Piazzarollo et al. (2019) para edifícios situados em Brasília.

\section{Conclusões}

O perfil de degradação existente nas fachadas localizadas no Distrito Federal pode ser observado pelas probabilidades de ocorrência de danos nas zonas da fachada. Os resultados do indicador de degradação Fator de Danos obtidos pela aplicação do Método de Mensuração de Degradação (MMD) indicam que mesmo com as variações de intensidade a zona com maior probabilidade de ocorrer degradação referemse às paredes contínuas e aos cantos e extremidades. Já a zona com menor quantidade de degradação foi as sacadas. Dentre os danos considerados em sistemas de revestimento cerâmico de fachadas, o descolamento cerâmico foi o dano mais evidente e com maior frequência de ocorrência. Esta pesquisa procura contribuir com os estudos e elaboração de novos projetos, buscando promover melhor 
compreensão dos fenômenos de degradação das fachadas o que auxilia na prevenção de danos bem como para indicar as zonas que necessitam de maior atenção e de ações de manutenção mais frequentes.

\section{Agradecimentos}

Os autores agradecem o apoio da Universidade de Brasília e a disponibilização das informações fornecidas pelo grupo de pesquisa do programa de pós-graduação em estruturas e construção civil da Universidade de Brasília que investiga degradação em fachadas.

\section{Referências Bibliográficas}

Bauer, E.; Castro, E. K.; Silva, M. N. B. (2015). Estimativa da degradação de fachadas com revestimento cerâmico: estudo de caso de edifícios de Brasília.CERÂMICA, v. 61, p. 151-159.

Bauer, E.; Piazzarollo, C. B.; Souza, J. S.; dos Santos, D. G. (2020). Relative importance of pathologies in the severity of facade degradation. Journal of Building Pathology and Rehabilitation, 5(1), 1-10.

Piazzarollo, C. B.; Souza, J. S.; Bauer, E. (2019). Souza, J. S. Análise das anomalias em sistemas de revestimento cerâmico nas diferentes zonas da fachada. XIII Simpósio Brasileiro de Tecnologia das Argamassas - SBTA 2019, Goiânia, Brasil; junho 2019.

Silva, F. G. S. (2006). Proposta de Metodologias Experimentais Auxiliares à Especificação e Controle das Propriedades Físico-Mecânicas dos Revestimentos em Argamassa. Dissertação de Mestrado em Estruturas e Construção Civil. Departamento de Engenharia Civil e Ambiental. Universidade de Brasília, Brasília, Brasil.

Silvestre, J. (2005). Sistema de Apoio à Inspeção e Diagnóstico de Anomalias em Revestimentos Cerâmicos Aderentes. Dissertação de Mestrado - Instituto Superior Técnico, Universidade Técnica de Lisboa, Lisboa, Portugal.

Sousa, R. D. (2008). Previsão da vida útil dos revestimentos cerâmicos aderentes em fachada. Dissertação de Mestrado, Universidade técnica de Lisboa Instituto Superior Técnico, Lisboa, Portugal.

Souza, J. S. (2016). Evolução da degradação de fachadas - efeito dos agentes de degradação e dos elementos constituintes. Dissertação de Mestrado. Universidade de Brasília, Brasília, Brasil.

Souza, J. S.; Piazzarollo, C. B.; Bauer, E.(2019a). Avaliação crítica do processo de degradação em sistemas de revestimento cerâmico de fachadas. International Congress about Pathology and Structures Rehabilitation - CINPAR 2019, Salta, Argentina; outubro 2019.

Souza, J. S.; Piazzarollo, C. B.; Bauer, E.(2019b).Degradação nas diferentes zonas de fachadas em sistemas de revestimento. XIII Simpósio Brasileiro de Tecnologia das Argamassas - SBTA 2019, Goiânia, Brasil; junho 2019.

Souza, J. S.; Piazzarollo, C. B.; Bauer, E.(2019c). Aplicação do método de mensuração de degradação em revestimentos de fachadas. II Workshop de Tecnologia de Processos e Sistemas Construtivos - TECSIC 2019, São Paulo, Brasil; agosto 2019. 